\title{
FECAL CALPROTECTIN DETERMINATION IN PRETERM NEONATES: EVALUATION OF TWO METHODS
}

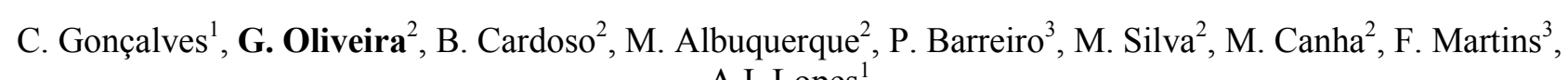
A.I. Lopes ${ }^{1}$

${ }^{1}$ Pediatric Gastrenterology Unit, ${ }^{2}$ Pediatric Department - Neonatology Service, University Hospital Santa Maria, ${ }^{3}$ Infectious Diseases, INSA, Lisbon, Portugal

Background and aims: Fecal calprotectin (FC) is a recognized marker of intestinal inflammation, previously validated in different clinical settings. Recent studies have suggested its uselfulness in early detection of neonatal necrotizing enterocolitis. Although standard methodology for FC determination is ELISA, quicker alternative techniques have been recently proposed, but not yet evaluated at this age group. A preliminary comparative analysis of FC concentration using ELISA and QuantumBlue* in preterm neonates was thus performed.

Methods: Fecal samples were prospectivelly collected postnatally at: 48-96hours (T1), end of 1st (T2) and 4th week of life (or at discharge) (T3), frozen at $-20^{\circ} \mathrm{C}$ and subsequently processed for determination of FC concentration using simultaneously Quick test (QuantumBlue ${ }^{\circledR}$ ) and ELISA (Buhlmann*)(two determinations using the two methods for each sample). Clinical data were also obtained. Descritive statistical analysis and Spearman correlation coefficient were performed.

Results: 14 neonates had a median gestacional age 32 weeks (25-34), birth weight:1636,7 $\pm 428,6$ grs; 2 cases with GI disease; number of samples assessed: $1 / 2,2 / 5,3 / 8$ neonates. The mean FC concentration $(\mathrm{mcg} / \mathrm{g})$ at T1 $(\mathrm{n}=24)$ was, respectively $: 257,93 \pm 191,87$ (quick test); $285,21 \pm 227,61$ (ELISA); at $\mathrm{T} 2:(\mathrm{n}=12)$ : $314,33 \pm 219,90$ (quick test); 357,33 $\pm 218,78$ (ELISA); at T3 ( $=8): 359,43 \pm 121,66$ (quick test); $349,29 \pm 191,87$ (ELISA). An overall 97.8\% correlation (p:0.001) between the two tests was found.

Conclusions: FC values found in this preliminary cohort of preterm neonates have been similar to those reported in the literature. The finding of a good correlation between the two techniques suggests the potential clinical usefulness of QuantumBlue at this age group (after validation). 